\title{
Evaluation of the Coherent Combining Lasers Array by the Power in the Bucket of the Main Lobe Method
}

\author{
Yang Liu, Xi Zhang, Debao Zhang, Wenqin Huang, Chang'an Qiao, Bo Wang \\ Wuhan Mechanical Technology College, Wuhan, China \\ Email: lyssym@126.com
}

Received 25 April 2016; accepted 19 August 2016; published 25 August 2016

\begin{abstract}
Coherent combination of laser beam is an important and challenging area of high power laser science. And how to evaluate the high power laser by coherent beam combination is a new research spot. Formulas for the radiated intensity distributions of coherent combined Gaussian beam array are derived via Fraunhofer scalar diffraction model by utilizing representations of the cross-spectral density of the far field. Effects of beam array numbers and separate distances etc. on far field radiated profiles are shown and analyzed. A new conception named power in the bucket of the main lobe $\left(P I B_{m}\right)$ is advanced to measure the beam quality of combined beams. This evaluation method is useful for efficiently determining the peak irradiance and power in the bucket for single emitting apertures of general shape.
\end{abstract}

\section{Keywords}

\section{Coherent Combination, Beam Shape, Fill Factor, Power in the Bucket}

\section{Introduction}

Achieving high optical power and high brightness has always been the major goals in the development of laser technologies. A natural means of increasing the power and especially the brightness should be to combine many individual lasers into arrays. In recent years, coherent and incoherent combinations of laser beams have been intensively studied theoretically and experimentally due to their wide applications in long-range energy delivering, etc. Since then, beam quality and divergence angle of laser arrays in the far field have also been investigated, and how to evaluate the high power laser by coherent beam combination is a new research hotspot [1] [2].

In this paper, based on the representations of the cross-spectral density of the far field, the radiated intensity distributions of coherent and incoherent combined Gaussian laser arrays are formulated and depicted. Effects of beam array numbers and separate distances between each source element on far-field radiated profiles are illustrated by numerical examples.

The important motivation for combining multiple lasers is to obtain high on-axis peak beam intensity, so the combination effect is evaluated by combining effect factor defined as the power in the bucket of the main lobe 
$\left(P I B_{m}\right)$ at the far field target. The $P I B_{m}$ is defined as

$$
P I B_{m}=\iint I(x, y) \cdot \operatorname{circ}\left(\frac{\sqrt{x^{2}+y^{2}}}{R}\right) d S / \iint I(x, y) d S
$$

$P I B_{m}$ is the ratio of the power in the main lobe to the total power of the array in the far filed intensity distribution. On this basis, a new conception is advanced to measure the beam quality of combined beams.

\section{Theory Model}

According to Fraunhofer diffraction theory, the diffraction field that collimated beam passing through lens can be calculated by Fraunhofer diffraction formula. In this paper, we established a laser combination array of several individual Gaussian beams with a near-field intensity profile arranged of a hexagonally packed group of emitters on a circle in a "bolt-hole" beam pattern as shown in Figure 1, and the far field beam profile calculation is based on scalar diffraction theory. The far field complex amplitude distribution is sensitive to near field beam relative phase relationship; array areal fill factor and array number [3]-[5]. We assume that every beam in the array has same intensity distribution with different beam center positions, and every beam waist width is equal as $\omega_{0}$, the distance between nearest beams is $d$, the total number of the laser array is $N, N=1+\sum_{m=1}^{M} 6 m, m$ is the circle number of the array.

The coordinate location of each beam can be referred as $\left(x_{m n}, y_{m n}\right)$,

$$
x_{m n}=m d \cdot \cos \left[(n-1) \frac{2 \pi}{6 m}\right], \quad y_{m n}=m d \cdot \sin \left[(n-1) \frac{2 \pi}{6 m}\right]
$$

The intensity of the laser array in the near field pattern can be written as

$$
\begin{aligned}
\boldsymbol{U}(x, y) & =\sum_{m=0}^{M} \sum_{n=1}^{6 m} A_{m n} \exp \left[-\frac{\left(x-x_{m n}\right)^{2}+\left(y-y_{m n}\right)^{2}}{\omega_{0}^{2}}+i \phi_{m n}\right] \\
& =\sum_{m=0}^{M} \sum_{n=1}^{6 m} A_{m n} \exp \left(-\frac{x^{2}+y^{2}}{\omega_{0}^{2}}+i \phi_{m n}\right) * \delta\left(x-x_{m n}, y-y_{m n}\right)
\end{aligned}
$$

where, $A_{m n}, \phi_{m n}$ is the complex amplitude and initial phase of each beam respectively. The far field profile is the spatial Fourier transform of the near field pattern, and the far field complex amplitude of coherent combined Gaussian laser array can be expressed as

$$
\begin{aligned}
& \boldsymbol{U}\left(f_{x^{\prime}}, f_{y^{\prime}}\right)=\frac{\exp \left(j k z^{\prime}\right)}{j \lambda z^{\prime}} \exp \left[j k\left(\frac{x^{\prime 2}+y^{\prime 2}}{2 z^{\prime}}\right)\right] \\
& \times \int_{-\infty}^{+\infty} \int_{-\infty}^{+\infty} \sum_{m=0}^{M} \sum_{n=1}^{6 m} A_{i j} \exp \left(-\frac{x^{2}+y^{2}}{\omega_{0}^{2}}+i \phi_{m n}\right) * \delta\left(x-x_{m n}, y-y_{m n}\right) \times \exp \left[-i 2 \pi\left(f_{x} x+f_{y} y\right)\right] d x d y
\end{aligned}
$$

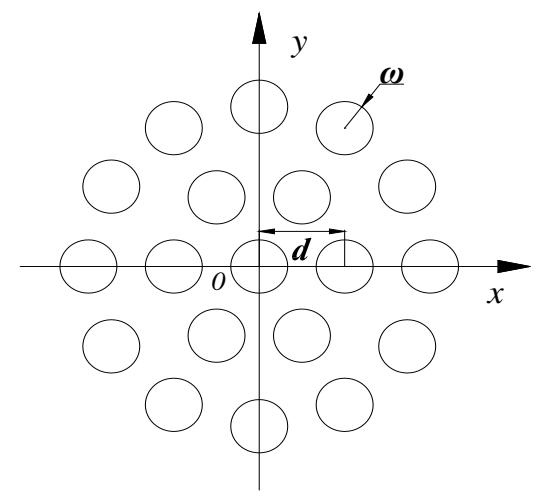

Figure 1. Arrangement of array beams. 
The far field cross-spectral intensity distribution pattern is the square of the complex amplitude model. For simplicity, we neglected the constant items, and the far field intensity distribution pattern of the array is easy to get as

$$
I\left(\theta_{x}, \theta_{y}\right) \propto\left|\sum_{m=0}^{M} \sum_{n=1}^{6 m} A_{m n} \exp \left\{i\left[k\left(x_{m n} \theta_{x}+y_{m n} \theta_{y}\right)-\phi_{m n}\right]\right\}\right|^{2} \times \exp \left[-\frac{k^{2} \omega_{0}^{2}}{2}\left(\theta_{x}^{2}+\theta_{y}^{2}\right)\right]
$$

where $\theta_{x, y}=m \lambda / d$, and we will see that the far field cross-spectral intensity distribution pattern is the product of the intensity of the laser array in the near field pattern with a single Gaussian beam. We induce a spatial coherence degree factor $\gamma$ which is defined as a fuzzy function of phase difference, complex amplitude, and polarization etc. Note that, the value of the coherence degree factor $\gamma$ is ranged from 0 to 1 . When $\gamma=1$, it refers that the array is completely coherent combined and when $\gamma=0$, the array is completely incoherent combined. In this paper, what we consider is the relative distribution of light intensity on far field, so we investigated the sensitive factors of array arrangement, coherence degree factor and the laser beam number which may influence the far field intensity distribution pattern. According to the above Formulas (2)-(5), we can simulate the far field distributions of the laser array and present the concept of $P I B_{m}$ to evaluate the coherent combining system's performances.

\section{The Far Filed Characters upon Sensitive Factors}

According to Fraunhofer diffraction theory, the diffraction field that collimated beam passing through lens can be calculated by Formula (5), where $i$ is imaginary unit, $k$ is wave number $\left(x_{m n}, y_{m n}\right)$ is the incident field coordinates, $\left(\theta_{x}, \theta_{y}\right)$ is the angle away from the normal, $N$ is the total number of wavelets, $d$ is the diameter of circular beams. For convenience, we demonstrate every sensitive factor assuming the other factors are constant.

\subsection{The Array Arrangement}

In this section, we study the performance of the array arrangement to the far field distribution. Suppose that there are only static phase distortions due to the laser path length differences between the different channels. Numerical calculations will be carried out to correct those phase distortions by finite number of iterations. We will consider hexagonal distributed laser arrays containing seven lasers as an example. We suppose that each fiber laser beam has a single Gaussian mode output. The beam waist of each laser is $\omega_{0}$, and lasers are arranged in an array with the nearest neighbor separated by a distance $d$. Vacancy factor $f=\left(2 \omega_{0} / d\right)$ can be used to describe the compactness of the array. A smaller $f$ corresponds to a more compact array. The numerical calculation of the far field distribution with variable $f=1.0,0.85,0.5,0.25$ is shown in Figure 2 .

From results it is found that, for coherent combination of Gaussian laser array, its intensity distributions would turn out to be occupy many rough lobes around the a central lobe. For example, when $f=1.0$, the far field distribution is the Gaussian type. With $f$ increases, more side-lobes appear which are induced by effects of interference of many separate source elements. While the vacancy factor decreased, the number of circle fringes increased but the radius of the central far-field lobe also decreased. What is most important is that the power in the bucket of the main lobe made the same corresponding changes. For certain $f$, the radius of the central far-field lobe can be calculated and the power in the bucket of the main lobe is the integration of such radius in the power domain.

\subsection{The Coherence Degree}

We induce a spatial coherence degree factor $\gamma$ which is defined as a fuzzy function of phase difference, complex amplitude, and polarization etc. [6]. From the Formula (5), it is easy to know that the factor $\gamma$ has no contributions to the far filed distribution pattern, but it is sensitive to the on axis density and the power of the main lobe. Assuming laser arrays containing seven lasers and its vacancy factor $f=0.8$, the numerical calculation of the far field transverse plane distribution with variable $\gamma$ value is shown in Figure 3 .

The computed results indicate that the radius of the main lobe of the far field distribution is consistent despite the variation of coherence degree factor $\gamma$. For a laser array with the coherence degree from $0-1$, the axial intensity vary approximately linearly with coherence, but the radius of the central far-field lobe is nearly unchanged as coherence decreases from one to nearly zero at which point the lobe disappears completely. Which 

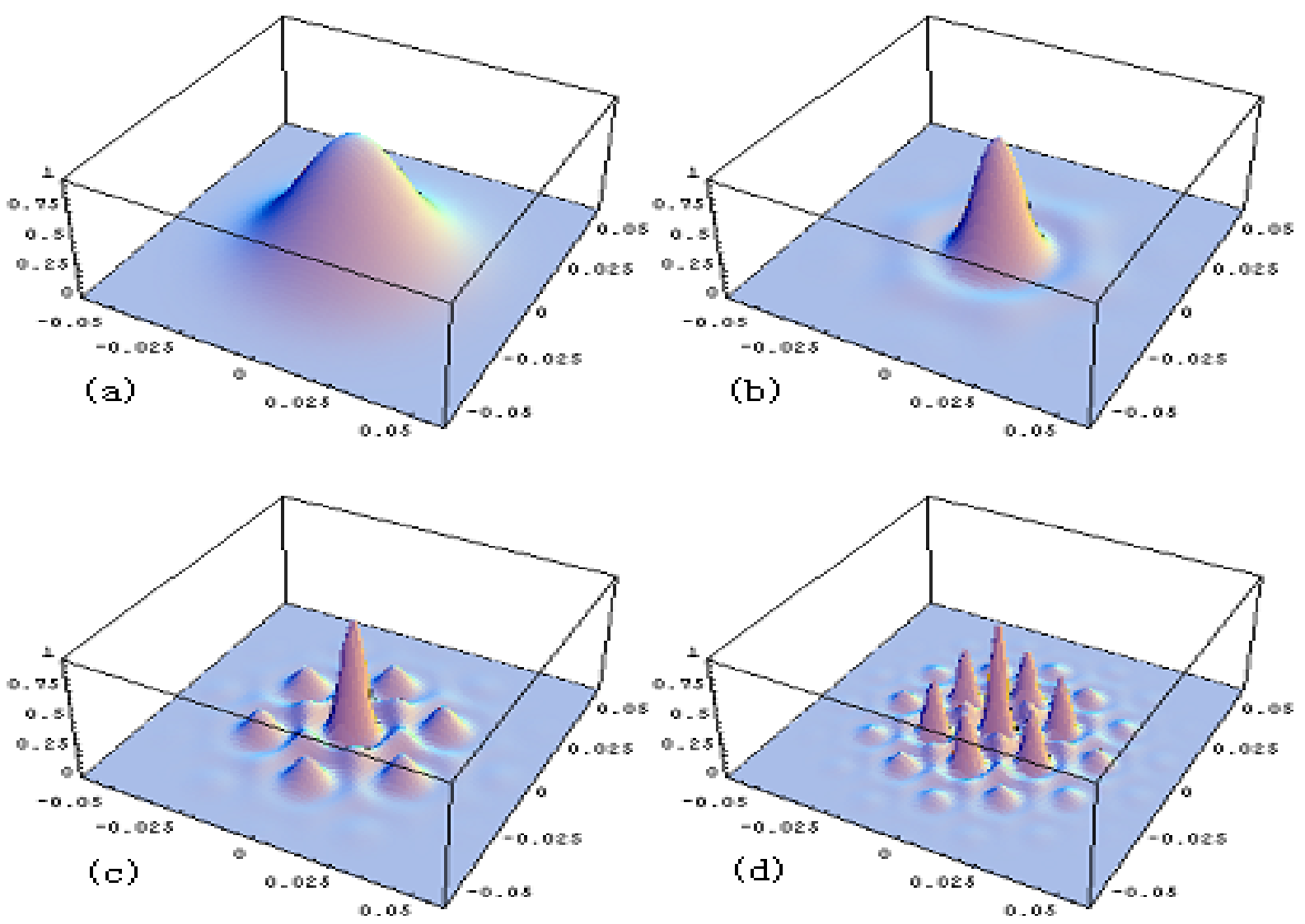

Figure 2. Far field distribution with various $f$ : (a) $f=1.0$ (b) $f=0.8$ (c) $f=0.5$ (d) $f=0.25$.

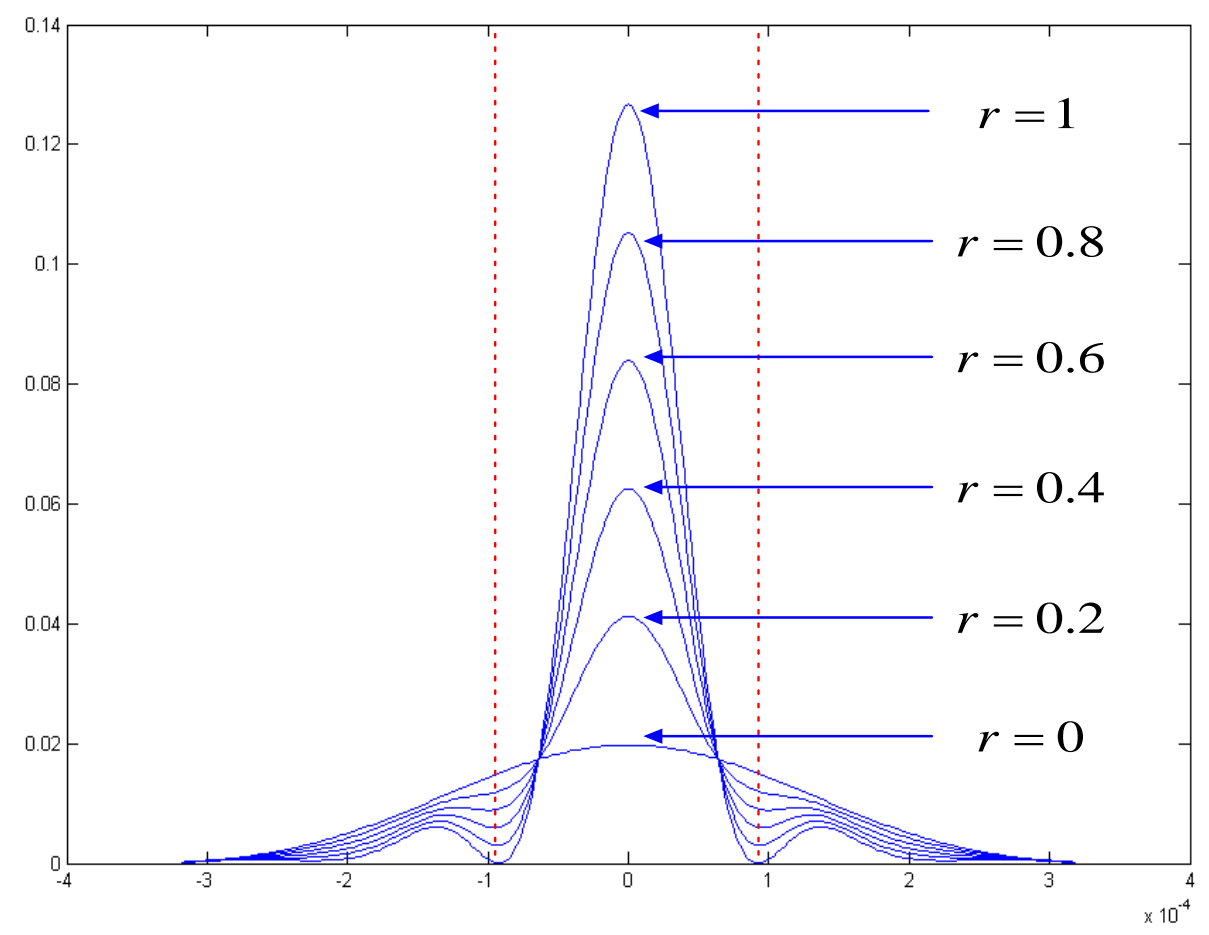

Figure 3. Far field distribution with various $\gamma$. 
may be the most important character is that, the fringe visibility at the far field does depend on the coherence degree but that dependence varies strongly with the array arrangement and the number of laser beams in the array.

\subsection{The Number of Array Beams}

The equations are an exception to the prescribed specifications of this template. You will need to determine whether or not your equation should be typed using either the Times New Roman or the Symbol font (please no other font). Equations should be edited by Mathtype, not in text or graphic versions. You are suggested to use Mathtype 6.0 (or above version).

From the Formula (5) of the theory model, total number of wavelets of the combination array does some effect to the far field intensity [7]. In this section, we computed the power of the main lobe versus the variable number of beams at a certain value of $f$. Figure 4 shows the calculated $P I B_{m}$ versus $m$ when $f=1.0,0.8$ and 0.5 independently.

In the graph, $m$ is the circle number of the array arrangement and the total number of the array is $N=1+\sum_{m=1}^{M} 6 m$. For example, if $m=0$, the total array number is 1 , if $m=5$, the total number is 91 . It is clear that, the effect to the $P I B_{m}$ by $m$ is very small, and the $P I B_{m}$ is fluctuating high and low irregularly with the variation of $m$. The fluctuating range is so limited that it even can be ignored compared with the effect of the array arrangement. Figure 5 gives the near and far field intensity distribution of array arrangement and the calculated $P I B_{m}$ value when $m=1,3,5$. For rough evaluation of coherent laser array combination, the computed $P I B_{m}$ seems to have little difference within $0.5 \%$.

\section{Power in the Bucket of the Main Lobe}

In this section we discuss the way how to rough evaluate the coherent beam combination by the method of power in the main lobe of the array's far field distribution. From the above analysis in Section 2 and Section 3, we know that for a given laser array, its far filed intensity distribution is determined by the factors of $f, \gamma, m$. For the far field intensity distribution shape, the main lobe almost consistent if the $f$ is arranged. The $P I B_{m}$ of the array is the ratio of the power in the main lobe to the total power of the array in the far filed intensity distribution.

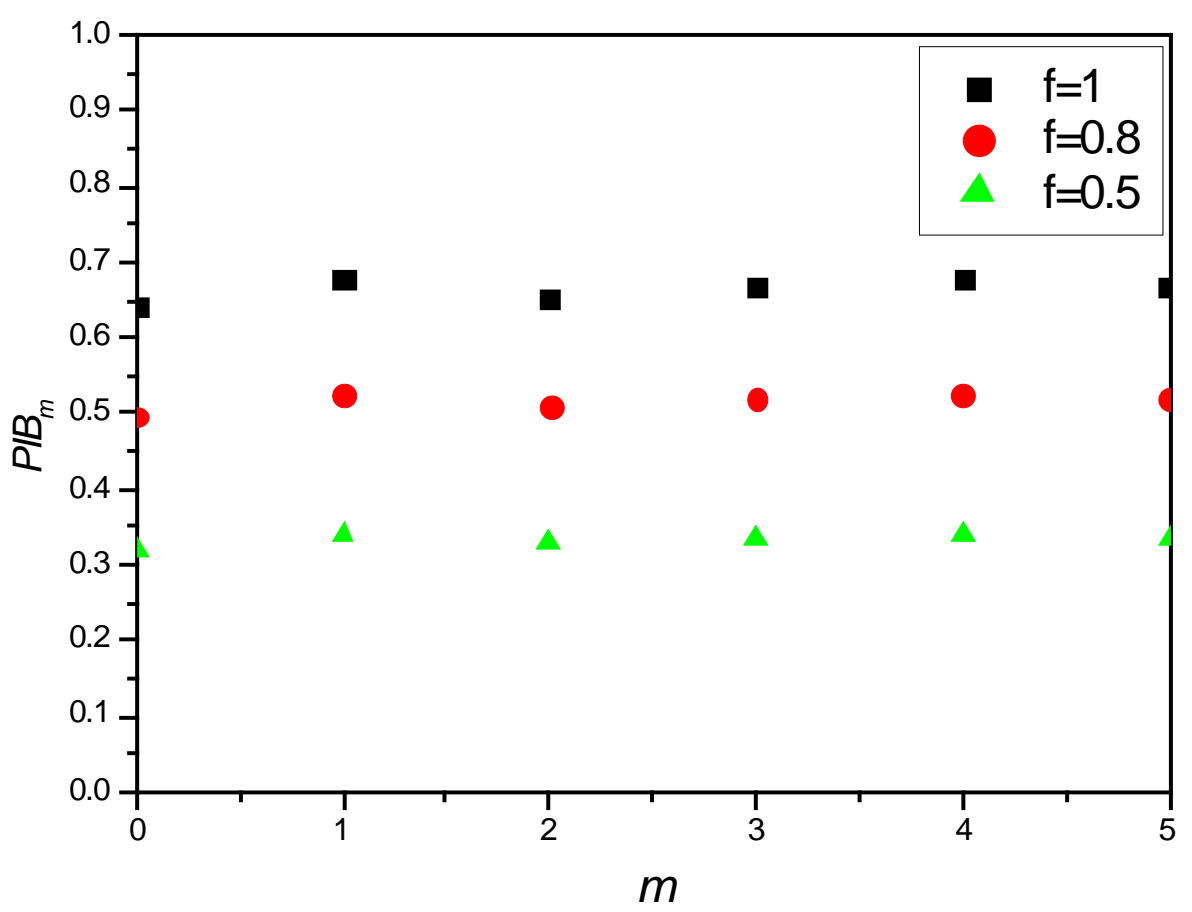

Figure 4. $P I B_{m}$ versus various $m$ at certain $f$. 

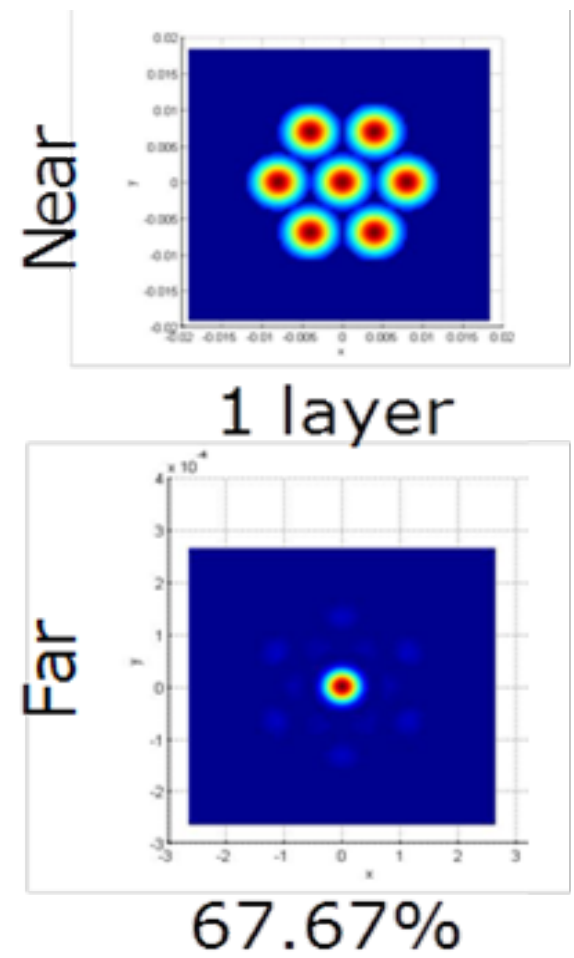
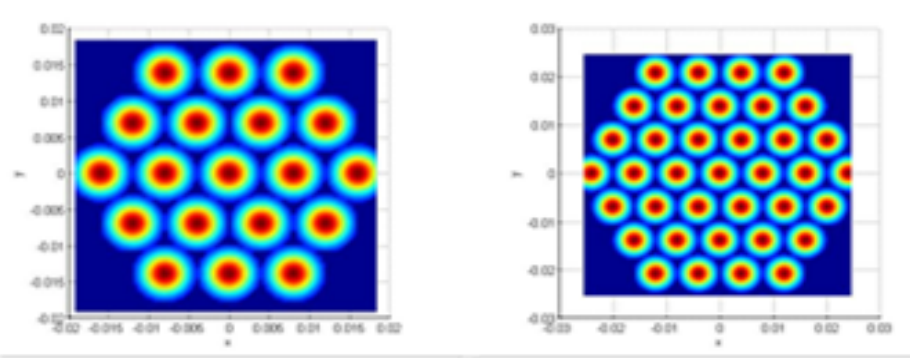

3 layers

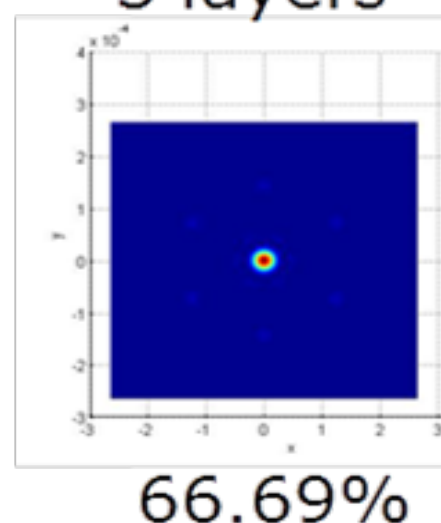

5 layers

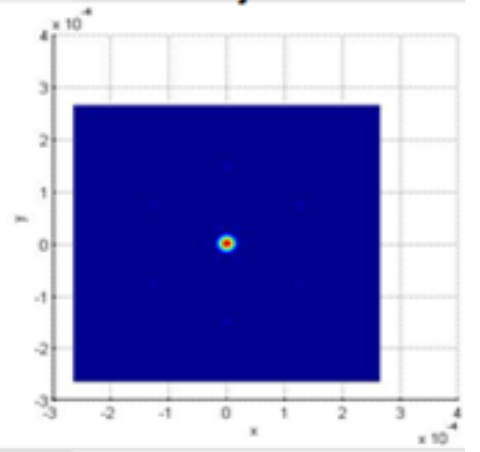

$66.93 \%$

Figure 5. Near and far filed distribution and the $\mathrm{PIB}_{\mathrm{m}}$ value when $\mathrm{m}=1,3,5$.

By the Formulas (4) and (5), we can calculate the $P I B_{m}$ in the ideal coherent combination state, and then take trap-hole method to measure the actual power in the bucket, the ratio of the actual power to the ideal calculated power is useful to evaluate the coherent combination effect of the sub lasers [8] [9]. A higher ration corresponds to a good coherent combination array. Laser's coherent combining is focused in high-energy laser field because of its increasing focal spot power density in evidence. Traditional evaluation criterion of beam quality can't represent predominance of coherent combining over incoherent combining. According to character of coherent combining, this $P I B_{m}$ method we presented for evaluation system of beam quality is constructed. This new evaluation system can represent significant predominance of coherent combining and improvement of laser's coherent combining system's performance.

\section{Conclusion}

In this paper, we have presented formulas for the radiated intensity distributions of coherent combined Gaussian beam array via Fraunhofer scalar diffraction model. We then deduced a set of formulae useful for efficiently determining the peak irradiance and power in the bucket for single emitting apertures of hexagonal distributed laser arrays. The far-field intensity distribution for coherent combination is numerically calculated and the sensitive factors are discussed in detail. A new conception named power in the bucket of the main lobe $\left(P I B_{m}\right)$ is proposed to measure the beam quality of combined beams. This evaluation method is useful for efficiently determining the peak irradiance and power in the bucket for single emitting apertures of general shape. Compared with other beam quality parameters, $P I B_{m}$ has many advantages on physical concept, feasibility and measurement in the project.

\section{References}

[1] Fan, T.Y. (2005) Laser Beam Combining for High-Power High Radiance Sources. IEEE J. Quantum. Electron., 11, 567-577. http://dx.doi.org/10.1109/JSTQE.2005.850241

[2] Li, Y.Z., Qian, L.J., Lu, D.Q., Fan, D.Y., Wen, S.C. (2007) Coherent and Incoherent Combining of Fiber Array with Hexagonal Ring Distribution. Opt Laser Tech, 39, 957-63. http://dx.doi.org/10.1016/j.optlastec.2006.06.010

[3] Zhou, Y., Liu, L.P., Peng, Q.J., et al. (2005) Phase Locking of Laser Array. SPIE, 6028, 602803. 
[4] Evans, G.A. and Hammer, J.M. (1993) Surface Emitting Semiconductor Lasers And Arrays. Academic Press, New York.

[5] Nabors, C.D. (1994) Effects of Phase Errors on Coherent Emitter Arrays. Appl Opt, 33, 2284-2289. http://dx.doi.org/10.1364/AO.33.002284

[6] Renwick, S.P. (2005) What Makes a Coherence Curve Change? Proc. of SPIE, 5754, 1537-1547. http://dx.doi.org/10.1117/12.598939

[7] Li, J., Chen, Y.R., Xu, S.X., et al. (2009) Far-Field Radiation of Coherent and Incoherent Combined Lorentz-Gaussian Laser Array. Proc. of SPIE, 7749, 1-6

[8] Smith, I.W. (2011) Beam Shape of Coherently-Combined Optical Arrays. Proc. of SPIE, 8052, 1-15. http://dx.doi.org/10.1117/12.883402

[9] Tan, Y. and Li, X.Y. (2012) Numerical Analysis of Beam Quality Factor $\beta$ on Coherent. Proc. of SPIE, 8551, 85511-1-9.

\section{Submit or recommend next manuscript to SCIRP and we will provide best service for you:}

Accepting pre-submission inquiries through Email, Facebook, Linkedin, Twitter, etc A wide selection of journals (inclusive of 9 subjects, more than 200 journals)

Providing a 24-hour high-quality service

User-friendly online submission system

Fair and swift peer-review system

Efficient typesetting and proofreading procedure

Display of the result of downloads and visits, as well as the number of cited articles

Maximum dissemination of your research work

Submit your manuscript at: http://papersubmission.scirp.org/ 